\title{
Skeletal muscle mass and sarcopenia in nonalcoholic fatty liver disease
}

\author{
Panadeekarn Panjawatanan ${ }^{1}$, Karn Wijarnpreecha ${ }^{2}$, Donghee Kim ${ }^{3}$ \\ ${ }^{1}$ Department of Internal Medicine, Bassett Medical Center, Cooperstown, New York, NY, USA; ${ }^{2}$ Division of Gastroenterology and Hepatology, \\ Mayo Clinic, Jacksonville, FL, USA; ${ }^{3}$ Division of Gastroenterology and Hepatology, Stanford University School of Medicine, Stanford, CA, USA \\ Correspondence to: Karn Wijarnpreecha. Division of Gastroenterology and Hepatology, Mayo Clinic, Jacksonville, FL, USA. \\ Email: dr.karn.wi@gmail.com. \\ Provenance: This an invited article commissioned by the Academic Editor Dr. Jia Zhu (Shenyang Pharmaceutical University, Shenyang, China). \\ Comment on: Cai C, Song X, Chen Y, et al. Relationship between relative skeletal muscle mass and nonalcoholic fatty liver disease: a systematic review \\ and meta-analysis. Hepatol Int 2019. [Epub ahead of print].
}

Received: 13 October 2019; Accepted: 31 October 2019; Published: 26 November 2019.

doi: $10.21037 /$ tgh.2019.11.03

View this article at: http://dx.doi.org/10.21037/tgh.2019.11.03

Nonalcoholic fatty liver disease (NAFLD) is currently the leading cause of chronic liver disease worldwide. Onefourth of the world population may have NAFLD, with higher prevalence in the Middle East and South America (1). In the US population, national estimates have shown that 80 million people were affected in 2015 , with the prediction of more than 100 million patients in 2030 (2). In Asia, the prevalence of NAFLD is as high as North America, which is thought to be due to the introduction of the Western diet. NAFLD has wide ranges of disease spectrum from nonalcoholic fatty liver, nonalcoholic steatohepatitis (NASH), and liver fibrosis, which can progress to cirrhosis and hepatocellular carcinoma. NAFLD is significantly associated with obesity, type 2 diabetes, hyperlipidemia, hypertension, and metabolic syndrome (1).

Sarcopenia is an age-related loss of muscle mass with impaired muscle strength and function. This condition affects approximately one-tenth of the world population and is projected to increase shortly as the world has been entering the aging society (3). Mechanism of sarcopenia involves aging processes like alteration of hormones, insulin resistance, cellular dysfunction, physical inactivity, malnutrition, and neurodegenerative diseases (4).

There are several essential studies attempted to find an association between sarcopenia and NAFLD, NASH, and advanced fibrosis in NAFLD. A Korean study found an increased risk of NASH and advanced fibrosis in the sarcopenic group, adjusted odds ratio (OR) 2.30 [95\% confidence intervals (CI): 1.08-4.93) and adjusted OR 2.05 (95\% CI: 1.01-4.16) respectively (5). A recent US population-based study showed a significant increase risk of NAFLD and advanced fibrosis after adjusted for metabolic risk factors and inflammatory markers, with adjusted OR 1.24 (95\% CI: $1.03-1.48)$ for NAFLD and adjusted OR 1.79 (95\% CI: 1.18-2.72) for advanced fibrosis (6). A recent longitudinal study in Korean population showed a benefit of increasing muscle mass in the incident and resolution of NAFLD, the adjusted hazard ratio (HR) 0.69 (95\% CI: $0.59-0.82)$ for the incidence of NAFLD and adjusted HR 4.17 (95\% CI: 1.90-6.17) for resolution of NAFLD (7). In terms of meta-analysis, a study by Wijarnpreecha et al. is the first paper to summarize all the published data (8). The result showed that sarcopenia increases the risk of NAFLD with a pooled OR 1.54 (95\% CI: 1.05-2.26), though there was high heterogeneity between studies (8). Sarcopenia also associated with histological NASH and advanced fibrosis in NAFLD from a meta-analysis of three studies, OR 2.35 (95\% CI: 1.45-3.81) and OR 2.41 (95\% CI: 1.94-2.98), respectively (9).

A recent meta-analysis from Cai et al. investigated the association between two diseases in terms of skeletal muscle mass (SMM) (10). In this study, skeletal muscle index (SMI) was calculated from SMM, which were obtained from two widely-used standard methods, bioimpedance analysis, and dual-energy X-ray absorptiometry. However, due to the absence of clear definition and cut-off value 
for the diagnosis of sarcopenia (11), SMM was adjusted with different parameters, including weight, height, and body mass index for calculation of SMI. But, in this metaanalysis, SMI was calculated based on adjusted SMM with bodyweight only, which eliminated the bias of different calculation methods. In this study, patients with NAFLD had low SMM compared to healthy control, with the weighted mean difference (WMD) of -1.77 (95\% CI: -2.39 to 1.15$)$. This result was consistent with the previous meta-analysis which showed an independent association between sarcopenia and NAFLD. However, there was still high heterogeneity between studies. In this study, subgroup analysis showed that women had lower WMD than men. This result is similar in a general population study, in which the prevalence of sarcopenia is equal among both genders, but the degree of sarcopenia tends to be more severe in women (12). There was an increased risk of NAFLD in sarcopenic population, with a pooled adjusted OR of 1.33 (95\% CI: 1.20-1.48), which is concordance with previous studies. The Asian population had a $37 \%$ increased risk of NAFLD compared to the Caucasian population. In terms of the association between sarcopenia and NASH, there is a significant association with a pooled OR of $2.42(95 \%$ CI: 1.27-3.57). The risk of significant fibrosis in sarcopenic patients has been determined with a pooled adjusted OR of 1.56 (95\% CI: 1.34-1.78) without any heterogeneity among studies.

Currently, liver biopsy is the gold standard for the diagnosis of NASH and advanced fibrosis. The non-invasive method such as elastography has some limitations in obese patients and in mild to moderate fibrosis (13). Another noninvasive method included FIB-4 and NAFLD fibrosis score to assess advanced fibrosis. The sensitivity and specificity for NAFLD fibrosis score is $67 \%$ and $97 \%$, while FIB-4 has a sensitivity of $34 \%$ and specificity of $98 \%$ (14). In this meta-analysis study, a subgroup analysis was performed based on different diagnostic methods of NAFLD (10). The result showed that there are higher odds for studies using invasive measures (liver biopsy) for diagnosing NAFLDrelated fibrosis among sarcopenia patients (OR 2.05, 95\% CI: 1.20-2.90) compared for studies using the non-invasive measures such as NFS, FIB-4 score (OR 1.52, 95\% CI: $1.29-1.75)(10)$.

This meta-analysis searched for studies in major databases with well-constructed search terms that covered the topic. Studies included were mostly high quality in study design and method, but few studies have low to moderate quality, which might affect heterogeneity and bias of the study. There was a high number of studies and study participants in the sarcopenia and NAFLD analytic group, which reflected the power of this meta-analysis, though there was some heterogeneity among the included studies. The diagnosis of sarcopenia is yet controversial due to variety in cut-off value and adjusted parameters which derived from the standard deviation of sex-specific mean skeletal mass in certain reference populations $(12,15)$. Because only two published studies were included for the Caucasian population compared to ten studies for Asians, this result regarding the association between sarcopenia and NAFLD needs to interpret cautiously and there should be more study to determine the risk in this population. Though there is significant publication bias in the report regarding the association between sarcopenia and NASH, the author proved that the bias did not affect the outcome by using the trim-and-fill method. However, there was still the minimal impact of the bias on the result after the trim-and-fill analysis, when they analyzed the association between sarcopenia and advanced fibrosis. Therefore, a cautious interpretation of the results is needed.

Postulated mechanisms for the association between sarcopenia and NAFLD may be explained by systemic inflammatory response, insulin resistance, and obesity (16). Cytokines and inflammatory markers including IL6 , TNF- $\alpha$, and CRP, are found in both patients with sarcopenia and NAFLD. These markers are contributed to the catabolic state of muscles, protein synthesis interference, and fat deposition in the liver (17). In addition, insulin resistance increases muscle protein breakdown and inhibits protein synthesis that contributes to muscle wasting and sarcopenia (18). Moreover, insulin resistance enhanced free fatty acid uptake and increased hepatic fat accumulation (19). Obesity is associated with sarcopenia and NAFLD due to the mechanism of insulin resistance and systemic inflammation modulated by adipokine production from adipose tissue (20).

Recently published studies, particularly from Asia, have shown similar outcomes of a relationship between sarcopenia and NAFLD, which affirms the result from this meta-analysis (21-23). Low handgrip strength as a proxy of muscle strength to define sarcopenia was significantly associated with NAFLD in men (OR 2.51, 95\% CI: 1.185.33), and women (OR 2.34, 95\% CI: 1.41-3.89), and was inversely correlated with hepatic steatosis index as a wellvalidated tool for NAFLD (24). However, a recent study from the Netherlands showed no association between sarcopenia and NAFLD in men, but the protective effect 
of SMM on NAFLD in women (25). Therefore, the race/ ethnicity might play some role in the association between two diseases.

Overall, this meta-analysis showed a comprehensive review of the association between SMM as a proxy of sarcopenia and NAFLD and significant fibrosis. There is a significantly increased risk of NAFLD and fibrosis in individuals with low muscle mass. In the future, more study is required to confirm the association in different ethnic groups and to understand more of pathophysiology between these two conditions.

\section{Acknowledgments}

None.

\section{Footnote}

Conflicts of Interest: The authors have no conflicts of interest to declare.

Ethical Statement: The authors are accountable for all aspects of the work in ensuring that questions related to the accuracy or integrity of any part of the work are appropriately investigated and resolved.

\section{References}

1. Younossi ZM, Koenig AB, Abdelatif D, et al. Global epidemiology of nonalcoholic fatty liver disease-Metaanalytic assessment of prevalence, incidence, and outcomes. Hepatology 2016;64:73-84.

2. Asrani SK, Devarbhavi H, Eaton J, et al. Burden of liver diseases in the world. J Hepatol 2019;70:151-71.

3. Shafiee G, Keshtkar A, Soltani A, et al. Prevalence of sarcopenia in the world: a systematic review and metaanalysis of general population studies. J Diabetes Metab Disord 2017;16:21.

4. Cruz-Jentoft AJ, Baeyens JP, Bauer JM, et al. Sarcopenia: European consensus on definition and diagnosis: Report of the European Working Group on Sarcopenia in Older People. Age Ageing 2010;39:412-23.

5. Koo BK, Kim D, Joo SK, et al. Sarcopenia is an independent risk factor for non-alcoholic steatohepatitis and significant fibrosis. J Hepatol 2017;66:123-31.

6. Wijarnpreecha K, Kim D, Raymond P, et al. Associations between sarcopenia and nonalcoholic fatty liver disease and advanced fibrosis in the USA. Eur J Gastroenterol
Hepatol 2019;31:1121-8.

7. Kim G, Lee SE, Lee YB, et al. Relationship Between Relative Skeletal Muscle Mass and Nonalcoholic Fatty Liver Disease: A 7-Year Longitudinal Study. Hepatology 2018;68:1755-68.

8. Wijarnpreecha K, Panjawatanan P, Thongprayoon C, et al. Sarcopenia and risk of nonalcoholic fatty liver disease: A meta-analysis. Saudi J Gastroenterol 2018;24:12-7.

9. Yu R, Shi Q, Liu L, et al. Relationship of sarcopenia with steatohepatitis and advanced liver fibrosis in non-alcoholic fatty liver disease: a meta-analysis. BMC Gastroenterol 2018;18:51.

10. Cai C, Song X, Chen Y, et al. Relationship between relative skeletal muscle mass and nonalcoholic fatty liver disease: a systematic review and meta-analysis. Hepatol Int 2019. [Epub ahead of print].

11. Batsis JA, Villareal DT. Sarcopenic obesity in older adults: aetiology, epidemiology and treatment strategies. Nat Rev Endocrinol 2018;14:513-37.

12. Janssen I, Heymsfield SB, Ross R. Low relative skeletal muscle mass (sarcopenia) in older persons is associated with functional impairment and physical disability. J Am Geriatr Soc 2002;50:889-96.

13. Wang JH. Application of Ultrasound Liver Elastography to the Diagnosis and Monitoring of Liver Disease. J Med Ultrasound 2019;27:1-2.

14. Cheah MC, McCullough AJ, Goh GB. Current Modalities of Fibrosis Assessment in Non-alcoholic Fatty Liver Disease. J Clin Transl Hepatol 2017;5:261-71.

15. Lee YH, Jung KS, Kim SU, et al. Sarcopaenia is associated with NAFLD independently of obesity and insulin resistance: Nationwide surveys (KNHANES 2008-2011). J Hepatol 2015;63:486-93.

16. De Fré CH, De Fré MA, Kwanten WJ. Sarcopenia in patients with non-alcoholic fatty liver disease: is it a clinically significant entity? Obes Rev 2019;20:353-63.

17. Zhai Y, Xiao Q. The Common Mechanisms of Sarcopenia and NAFLD. 2017;2017:6297651.

18. Cleasby ME, Jamieson PM, Atherton PJ. Insulin resistance and sarcopenia: mechanistic links between common comorbidities. J Endocrinol 2016;229:R67-81.

19. Kitade H, Chen G, Ni Y, et al. Nonalcoholic Fatty Liver Disease and Insulin Resistance: New Insights and Potential New Treatments. Nutrients 2017;9. doi: 10.3390/ nu9040387.

20. Khan M, Joseph F. Adipose tissue and adipokines: the association with and application of adipokines in obesity. Scientifica (Cairo) 2014;2014:328592. 
21. Chung GE, Kim MJ, Yim JY, et al. Sarcopenia Is Significantly Associated with Presence and Severity of Nonalcoholic Fatty Liver Disease. J Obes Metab Syndr 2019;28:129-38.

22. Gan D, Wang L, Jia M, et al. Low muscle mass and low muscle strength associate with nonalcoholic fatty liver disease. Clin Nutr 2019. [Epub ahead of print].

23. Zhang Y, Lu D, Wang R, et al. Relationship between Muscle Mass/Strength and Hepatic Fat Content in PostMenopausal Women. Medicina (Kaunas) 2019;55. doi:

doi: $10.21037 / \operatorname{tgh} .2019 .11 .03$

Cite this article as: Panjawatanan P, Wijarnpreecha K, Kim D. Skeletal muscle mass and sarcopenia in nonalcoholic fatty liver disease. Transl Gastroenterol Hepatol 2019;4:77. 10.3390/medicina55100629.

24. Kim BJ, Ahn SH, Lee SH, et al. Lower hand grip strength in older adults with non-alcoholic fatty liver disease: a nationwide population-based study. Aging (Albany NY) 2019;11:4547-60.

25. Alferink LJM, Trajanoska K. Nonalcoholic Fatty Liver Disease in The Rotterdam Study: About Muscle Mass, Sarcopenia, Fat Mass, and Fat Distribution. J Bone Miner Res 2019;34:1254-63. 